\title{
A Preterm Infant With Mild Abdominal Distension and Rising C-Reactive Protein
}

\author{
Atef Alshafei ${ }^{\mathrm{a}, \mathrm{b}}$, Mahmoud Ahmed ${ }^{\mathrm{a}}$
}

\begin{abstract}
Abdominal distension is a common clinical presentation in neonates admitted to neonatal intensive care units (NICUs), especially preterm infants. The underlying causes are multiple and range from simple transient etiologies to life-threatening events with significant morbidities. Neonatal sepsis is the most common diagnosis encountered; however, other differential diagnoses should be thought. Premature neonates are particularly susceptible to abdominal distension because of gut immaturity, relative immune deficiency and frequent exposure to invasive procedures. Herein, we report a preterm infant who developed abdominal distension and hepatomegaly on day 4 of life. This was associated with progressively rising $\mathrm{C}$-reactive protein (CRP) levels and normal abdominal X-ray. Bed side abdominal ultrasonography revealed the diagnosis.
\end{abstract}

Keywords: Abdominal distension; Neonate; CRP; Hepatomegaly

\section{Introduction}

Abdominal distension with hepatomegaly in preterm infants is a challenging clinical scenario with quite large number of differential diagnoses. However, systematic approach including proper history taking, physical examination and basic laboratory and radiological studies would potentially narrow the diagnostic list. Most of these infants are sick, receiving ventilation support and having indwelled central catheters and hence, infectious etiologies are of primary concern. Other differential diagnoses including inborn errors of metabolism, congenital infections, hepatic infiltration and biliary tract obstruction should be considered and high index of suspicion is required for timely diagnosis. This report emphasizes the significant role of bed side ultrasonography as a quick and invaluable diagnostic modality when utilized by a trained neonatologist in cases of abdominal distension.

Manuscript submitted January 25, 2019, accepted March 25, 2019

aNICU, Pediatric Department, Dubai Hospital, Dubai, United Arab Emirates ${ }^{b}$ Corresponding Author: Atef Alshafei, Neonatology Section, Dubai Hospital, PO Box 7272, Dubai, United Arab Emirates. Email: Ahalshafei@dha.gov.ae

doi: https://doi.org/10.14740/ijcp326

\section{Case Report}

A preterm male infant was born at an estimated $29+3 / 7$ weeks' gestation via normal vaginal delivery to a 30 -year-old gravida 3 , para $2+0$ woman. She had positive history of leaking for 5 days prior to delivery. Her urine, high vaginal swab cultures and serologies were negative.

The infant's birth weight was 1,420 $\mathrm{g}$ and Apgar scores are 8 and 9 at 1 and $5 \mathrm{~min}$, respectively. On examination, he was active, well perfused, and systemic examination revealed normal cardiovascular findings, no neurologic focal deficits and unremarkable abdominal examination. Vital signs showed temperature of 37 ${ }^{\circ} \mathrm{C}$, respiratory rate of $45-55$ breaths per minute, heart rate of 130 - 150 beats per minute, oxygen saturation of $95-99 \%$ and blood pressure of $60 / 40 \mathrm{~mm} \mathrm{Hg}$ on right hand by cuff method.

He was supported by nasal continuous positive air way pressure (nCPAP) of $6 \mathrm{~cm} \mathrm{H}_{2} \mathrm{O}$, and total parenteral nutrition (TPN) was initiated via umbilical venous catheter (UVC). X-ray of chest and abdomen was essentially normal (Fig. 1.) Complete blood count (CBC) was normal, and blood culture after admission showed negative results after receiving a 48 -h course of ampicillin and gentamycin. On day 2 after birth, C-reactive protein (CRP) was $7.1 \mathrm{mg} / \mathrm{L}$ (normal $<5 \mathrm{mg} / \mathrm{L}$ ) and antibiotics were continued. On day 4 , the infant had mild abdominal distension, but he was active, well perfused and tolerating breast milk feeds $40 \mathrm{~mL} / \mathrm{kg} /$ day. Abdominal examination revealed hepatomegaly $3 \mathrm{~cm}$ below right costal margin but no splenomegaly or ascites. Blood gases, urea, electrolytes, creatinine and liver function tests were normal (Table 1), but the repeated CRP increased to $22 \mathrm{mg} / \mathrm{L}$, then to $41.5 \mathrm{mg} / \mathrm{L}$. Antibiotics were changed at this stage to cloxacillin and ceftazidime and fresh frozen plasma was given in view of deranged coagulation profile. Baby was kept nil per os, and abdominal ultrasound was done by the attending neonatologist (Supplementary video 1, www.theijcp.org and Fig. 2). A multiloculated fluid collection with hyperechoic rim was seen in the right lobe of the liver measuring $4.1 \times 3.2 \mathrm{~cm}$. Mild hepatomegaly was observed but no ascites, and no obvious intra- or extrahepatic biliary radicle dilation was seen. Doppler study reported no significant vascularity within the focal lesion in liver. The adjacent hepatic and portal vein demonstrated normal color flow. X-ray of abdomen done at that time showed abnormally displaced umbilical venous catheter (UVC) below the diaphragm (Fig. 3). UVC was removed and replaced by a peripherally inserted central catheter (PICC). The differential diagnosis at this stage is for neonatal hepatic lesion with hypoechoic multiseptated center and hyperechoic rim and includes hemato- 


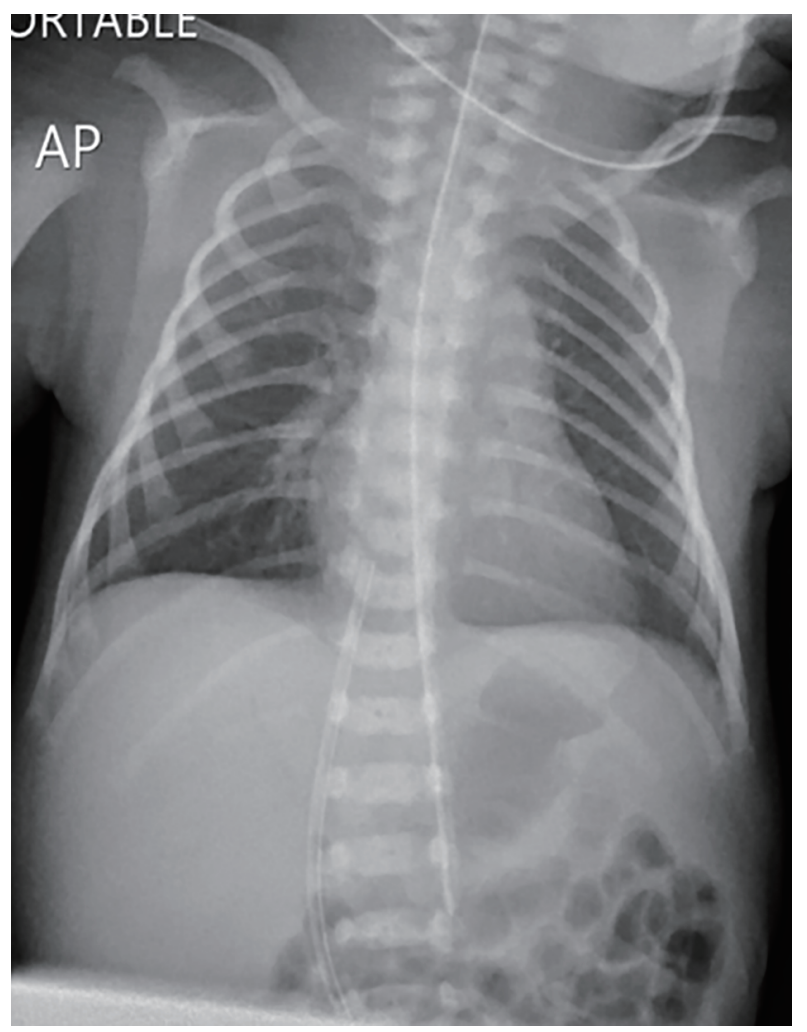

Figure 1. Normal chest and abdomen radiology on admission showing correctly placed UVC at the level of T9. UVC: umbilical venous catheter.

ma secondary to birth trauma, hemangio-endothelioma, hepatic abscesses and hepatic necrosis due to UVC extravasation.

\section{Diagnosis}

Although uncommon in neonates, hepatic erosion by UVC extravasation of TPN is the most likely etiology in our patient. This is based on the presentation of a preterm infant with hyperalimentation via indwelling UVC that is followed by hepa-

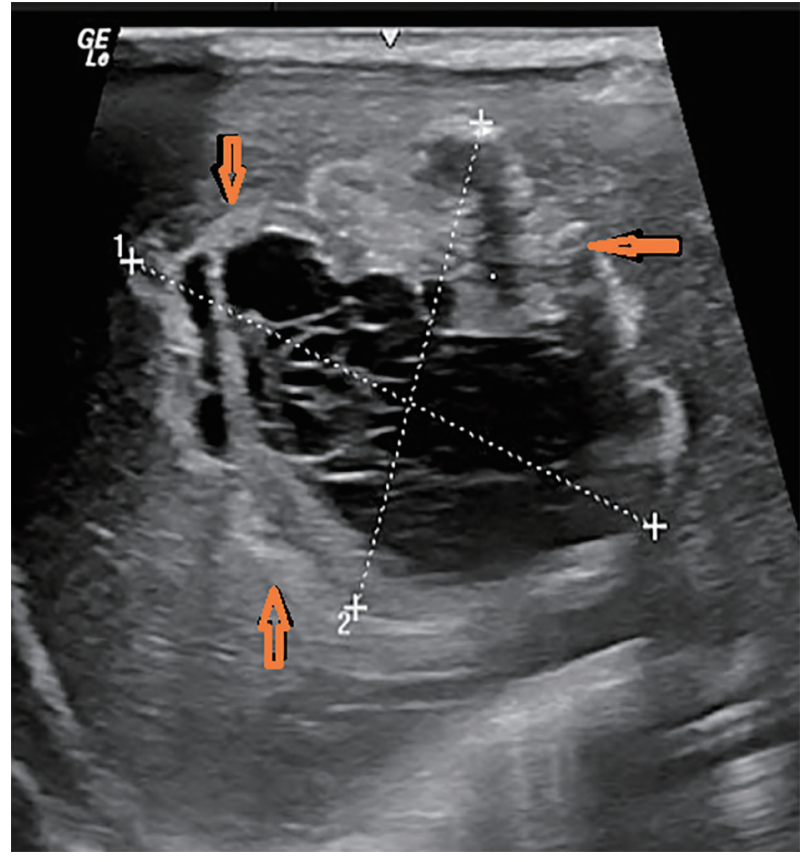

Figure 2. Abdominal ultrasound shows multiloculated cystic lesion in the right lobe of liver measuring $4.1 \times 3.2 \mathrm{~cm}$ (red arrows).

tomegaly with rising CRP. There is no history of traumatic labor to explain hematoma and the infant was hemodynamically stable with no drop-in hemoglobin. Furthermore, ultrasound findings are not suggestive of hepatic abscesses nor hepatic hemangioendothelioma. Hepatic abscess in neonates is a serious diagnostic challenge and usually presents as multiple abscesses with a fulminant septic course and poor outcome [1].

Pediatric surgeon and pediatric gastroenterologist reviewed the case and advised for conservative approach, continuing antibiotics, and gradually resuming enteral feeding. Feeds were tolerated and advanced uneventfully reaching full enteral intake at the age of 20 days. He received a 2 -week course of antibiotics and discharged at the age of 45 days weighing $2.1 \mathrm{~kg}$. The repeated serial abdominal ultrasound examinations revealed complete resolution of the cystic lesion,

Table 1. Laboratory Data Follow-Up on Admission Till 4 Months of Age

\begin{tabular}{|c|c|c|c|c|c|c|c|c|c|c|c|c|c|c|c|}
\hline & \multicolumn{3}{|c|}{ FBC } & \multicolumn{7}{|c|}{ LFT } & \multirow{2}{*}{ PT } & \multirow{2}{*}{ CRP } & \multirow{2}{*}{$\mathrm{Na}$} & \multirow{2}{*}{$\mathbf{K}$} & \multirow{2}{*}{$\mathrm{Cr}$} \\
\hline & WBC & $\mathbf{H b}$ & Plat & $\mathrm{TB}, \mathrm{mg} / \mathrm{dL}$ & ALP, U/L & ALT, U/L & $\mathrm{TP}, \mathrm{g} / \mathrm{dL}$ & Alb, g/dL & Glob & AFP, ng/mL & & & & & \\
\hline Day 1 & 13.2 & 14.9 & 372 & 0.9 & - & - & - & - & - & - & - & 7.1 & 139 & 5 & - \\
\hline Day 10 & 8.9 & 8 & 455 & 1.9 & 259 & 22 & 5.1 & 3 & 2.1 & 30,000 & 12 & 41.5 & 139 & 5.4 & 0.6 \\
\hline Day 24 & 8 & 9.7 & 487 & 1 & 277 & 13 & 5.3 & 2.9 & 2.4 & 30,000 & 10 & 29.9 & 137 & 4.1 & 0.5 \\
\hline 2 months & 8 & 8 & 561 & 0.6 & 411 & 14 & 4.8 & 3 & 1.8 & 11,156 & - & 5.4 & 137 & 4.9 & 0.5 \\
\hline 4 months & - & - & - & 1 & 440 & 15 & 4.8 & 3.2 & 1.6 & 1,258 & - & $\mathrm{Neg}$ & 138 & 4.7 & - \\
\hline
\end{tabular}

FBC: full blood count; LFT: liver function test; WBC: white blood cells; $\mathrm{Hb}$ : hemoglobin; Plat: platelets; TB: total bilirubin; ALP: alkaline phosphatase; ALT: alanine transferase; TP: total protein; Alb: albumin; Glob: globulin; AFP: alpha-fetoprotein protein; PT: prothrombin time; CRP: C-reactive protein; Cr: creatinine. 


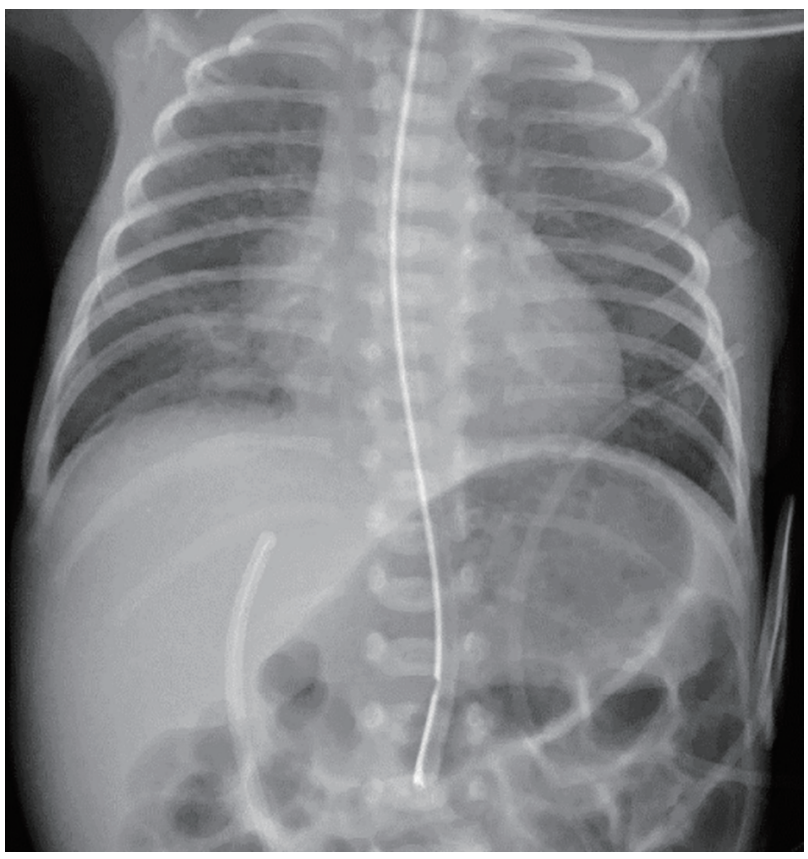

Figure 3. Abdominal X-ray on day 4 shows downward displacement of UVC in the hepatic parenchyma. UVC: umbilical venous catheter.

normal portal vein and no residual damage or complications (Fig. 4a-e). Serial liver function tests (LFTs) were normal and CRP levels were declining gradually as well. Sequential laboratory findings are summarized in Table 1. On follow-up at the age of 6 months, he was clinically well and achieving normal developmental milestones for age.

\section{Discussion}

In the patient described here, abdominal distension was the presenting sign of an under recognized clinical complication of UVC malposition. Although UVC was correctly inserted on admission and position was verified by radiology, yet significant TPN extravasation in hepatic parenchyma occurred on day 4. The onset of diagnosis in our patient was earlier than many reported cases of UVC extravasation that manifested usually beyond the first week of life [2-5]. The prompt diagnosis was augmented by the bed side ultrasonography as a simple noninvasive diagnostic tool that can be operated easily in NICU.

UVC placement is an invaluable procedure for parenteral nutrition and drug administration in sick neonates admitted to NICU [6]. Unfortunately, these catheters are associated with increased risk of short- and long-term complications including infection, hemorrhage, thrombo-embolic formation, catheter migration, cardiac arrhythmias and pleural and pericardial effusion.

Although neonatal sepsis is the commonest diagnosis to consider in a preterm infant with abdominal distension, indwelling venous catheter, positive history of premature rupture of membranes and rising inflammatory markers, yet other differential diagnoses should be considered. These include necrotizing enterocolitis (NEC), congenital infections, inborn errors of metabolism, hepatic tumors, biliary tract obstruction and congestive heart failure. Hepatic infiltration by UVC extravasation is a serious and uncommon condition that should be suspected as a potential complication of malpositioned UVC. Ideally, the tip
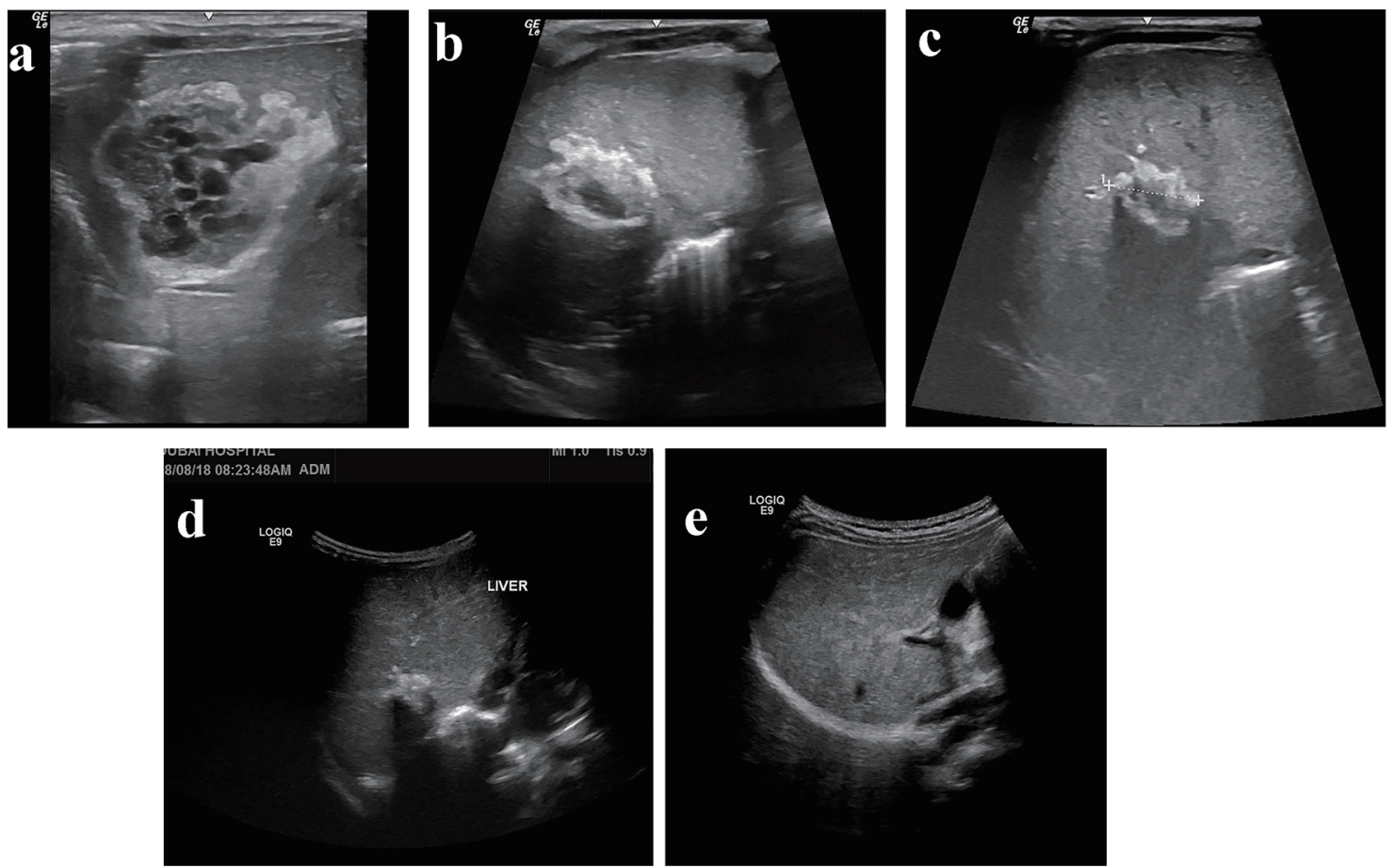

Figure 4. (a-e) Serial abdominal ultrasound images show the gradual regression of the hepatic collection with complete resolution at 6 months of age. 
of the UVC should lie at the junction of the inferior vena cava (IVC) and the right atrium or just above the diaphragm at the level of T7-T9 [7]. UVC can be easily misplaced from a previously normal position if not fixed well and meticulously observed. The rate of UVC malposition varies greatly and ranges from $20 \%$ to $37 \%$ [8]. Complications of malpositioned UVC are uncommon in neonates and include hepatic necrosis, collection, laceration and abscess formation [9, 10]. Significant extravasation of hypertonic solutions, TPN, or blood products may inadvertently leak into the hepatic parenchyma and cause significant hepatic injury. TPN is alkaline in nature and accidental leak may cause chemical irritation or parenchymal compression with significant hepatic necrosis if not promptly recognized.

Typically, patients develop clinical deterioration with hypotension, shock, pallor, abdominal distension and ascites. However, symptoms can be subtle, and a high index of suspicion is necessary for timely recognition and prevention of lifethreating complications [11]. Treatment of UVC extravasation is mainly supportive; however, surgical intervention may be necessary in some cases including paracentesis, peritoneal drain (Penrose) insertion or laparotomy. Prognosis is generally good with complete recovery of uncomplicated cases if the condition is promptly recognized and treated [12].

\section{Conclusions}

High index of suspicion is required to recognize early extravasation in an infant who is on TPN via UVC and then develops clinical deterioration including abdominal distension.

After catheterization, the position of UVC should be verified by X-ray or preferably by ultrasound. Meticulous observation and monitoring to avoid displacement are paramount and immediate removal of UVC is crucial if found to be malpositioned on radiography $[13,14]$.

Inflammatory markers including CRP are not always indications for sepsis, therefore, other diagnoses should be thought.

\section{Funding}

No specific support.

\section{Financial Disclosure}

Drs Alshafei and Ahmed have disclosed no financial relationships relevant to this article. This commentary does not contain a discussion of an unapproved/investigative use of a commercial product/device.

\section{Conflict of Interest}

The authors have no conflict of interest to disclose.

\section{References}

1. Moss TJ, Pysher TJ. Hepatic abscesses associated with umbilical catheterization in two neonates. Hepatic abscess in neonates. Am J Dis Child. 1981;135:726-728.

2. Sherwani P, Vire A, Anand R, Jajoo M. Umbilical venous catheterization gone wrong: Hepatic complications. Indian J Radiol Imaging. 2016;26(1):40-43.

3. Shareena I, Khu YS, Cheah FC. Intraperitoneal extravasation of total parental nutrition infusate from an umbilical venous catheter. Singapore Med J. 2008;49(2):e35-36.

4. Yeh J, Vargas J, Wozniak L, et al. Massive liver mass and parenteral nutrition extravasation secondary to umbilical venous catheter complications. Journal of clinical neonatology. 2014;3(3):158-160.

5. Lim-Dunham JE, Vade A, Capitano HN, Muraskas J. Characteristic sonographic findings of hepatic erosion by umbilical vein catheters. J Ultrasound Med. 2007;26(5):661-666.

6. Mutlu M, Aslan Y, Kul S, Yilmaz G. Umbilical venous catheter complications in newborns: a 6-year single-center experience. J Matern Fetal Neonatal Med. 2016;29(17):2817-2822.

7. Oestreich AE. Umbilical vein catheterization - appropriate and inappropriate placement. Pediatr Radiol. 2010;40(12):1941-1949.

8. Tsui BC, Richards GJ, Van Aerde J. Umbilical vein catheterization under electrocardiogram guidance. Paediatr Anaesth. 2005;15(4):297-300.

9. Abiramalatha T, Kumar M, Shabeer MP, Thomas N. Advantages of being diligent: lessons learnt from umbilical venous catheterization in neonates. BMJ Case Rep. 2016;2016:bcr2015214073.

10. Gulcan H, Hanta D, Torer B, Temiz A, Demir S. Hepatic laceration as a life-threatening complication of umbilical venous catheterization. Turk J Pediatr. 2011;53(3):342345.

11. Yigiter M, Arda IS, Hicsonmez A. Hepatic laceration because of malpositioning of the umbilical vein catheter: case report and literature review. J Pediatr Surg. 2008;43(5):E39-41.

12. Adesanya O, Naqvi M. Term Neonate With Liver Laceration, Obstructive Uropathy, and Ascites-Secondary to Extravasation of Total Parenteral Nutrition: A Complication of Malpositioned Umbilical Venous Catheter. Glob Pediatr Health. 2016;3:2333794X16670494.

13. Pulickal AS, Charlagorla PK, Tume SC, Chhabra M, Narula P, Nadroo AM. Superiority of targeted neonatal echocardiography for umbilical venous catheter tip localization: accuracy of a clinician performance model. J Perinatol. 2013;33(12):950-953.

14. Franta J, Harabor A, Soraisham AS. Ultrasound assessment of umbilical venous catheter migration in preterm infants: a prospective study. Arch Dis Child Fetal Neonatal Ed. 2017;102(3):F251-F255. 